\title{
ON THE NUMBER OF APPARENT TRIPLE POINTS OF SURFACES IN SPACE OF FOUR DIMENSIONS
}

\author{
BY B. C. WONG
}

Two hypersurfaces in 4-space of orders $\mu$ and $\nu$ respectively intersect in a surface $F$ of order $\mu \nu . F$ has a certain number $H$ of apparent triple points, that is, lines that can be drawn through a given point meeting $F$ three times. If $F$ degenerates into an $F_{1}$ of order $m_{1}$ and an $F_{2}$ of order $m_{2}$ where $m_{1}+m_{2}=\mu \nu$, then $H$ is the sum of the numbers $h_{30}, h_{21}, h_{12}$, $h_{03}$, where $h_{i j}$ is the number of lines that pass through a given point and meet $F_{1} i$ times and $F_{2} j$ times. It is the purpose of this paper to determine $H$ and, if $F$ is composite, to determine the distribution of the $h_{i j}$ lines.

The formula for $H$ can be readily obtained by calculating the order of the restricted system of equations resulting from imposing upon two binary equations of orders $\mu$ and $\nu$ respectively the conditions that they have three common roots. ${ }^{*}$ But this method does not offer a ready means for the determination of the distribution of the $h_{i j}$ lines if $F$ is composite. The following method seems well adapted for the purpose.

Suppose, temporarily, that the two hypersurfaces giving $F$ be composed of $\mu$ and $\nu$ hyperplanes $\alpha_{k}, \beta_{l}[k=1,2, \cdots, \mu$; $l=1,2, \cdots, \nu]$. Then $F$ is made up of $\mu \nu$ planes $\alpha_{k} \beta_{l}$. We construct the rectangular array

$$
\begin{aligned}
& \alpha_{1} \beta_{1} \quad \alpha_{2} \beta_{1} \quad \alpha_{3} \beta_{1} \cdots \alpha_{\mu} \beta_{1} \\
& \begin{array}{lll}
\alpha_{1} \beta_{2} & \alpha_{2} \beta_{2} & \alpha_{3} \beta_{2} \cdots \alpha_{\mu} \beta_{2}
\end{array} \\
& \begin{array}{lll}
\alpha_{1} \beta_{3} & \alpha_{2} \beta_{3} & \alpha_{3} \beta_{3} \cdots \alpha_{\mu} \beta_{3}
\end{array} \\
& \begin{array}{lll}
\alpha_{1} \beta_{\nu} & \alpha_{2} \beta_{\nu} & \alpha_{3} \beta_{\nu} \cdots \alpha_{\mu} \beta_{\nu}
\end{array}
\end{aligned}
$$

* Salmon, Modern Higher Algebra, 4th ed., Lesson 19. 
and interpret it as the symbolic representation of $F$, proper or improper. In this interpretation we remove the assumption that the hypersurfaces are composed of hyperplanes and the $\alpha_{k} \beta_{l}$ are to be regarded as mere symbols.

Each of the constituents of the array (A), taken alone, represents a plane. A pair of constituents represents a quadric surface or two incident planes if the constituents are in the same row as $\alpha_{1} \beta_{1}, \alpha_{2} \beta_{1}$ or in the same column as $\alpha_{1} \beta_{1}$, $\alpha_{1} \beta_{2}$; two non-incident planes if they are in different rows and columns from $\alpha_{1} \beta_{1}, \alpha_{2} \beta_{2}$. Three constituents in the same row as $\alpha_{1} \beta_{1}, \alpha_{2} \beta_{1}, \alpha_{3} \eta_{1}$ or in the same column as $\alpha_{1} \beta_{1}, \alpha_{1} \beta_{2}, \alpha_{1} \beta_{3}$ represent a cubic surface lying wholly in an $S_{3}$ : if the constituents are such that one of them lies in the same column with another and in the same row with the third as $\alpha_{1} \beta_{2}$, $\alpha_{1} \beta_{1}, \alpha_{2} \beta_{1}$, the cubic surface is a 4 -space surface. Three nonincident planes are represented by three constituents all in different rows and columns from $\alpha_{1} \beta_{1}, \alpha_{2} \beta_{2}, \alpha_{3} \beta_{3}$. Since from a given point only one line can be drawn meeting three nonincident planes each once, the presence of such a triple of constituents, all lying in different rows and columns, in the array means the presence of an apparent triple point on $F$. The total number of possible triples of this sort in $(A)$ is the total number of possible apparent triple points of $F$ and the formula for this number is evidently

$$
H=\mu \nu(\mu-1)(\mu-2)(\nu-1)(\nu-2) / 6 .
$$

Now if $F$ is composed of an $F_{1}$ of order $m_{1}$ and an $F_{2}$ of order $m_{2}$, the constituents of (A) are divided into two groups: one of $m_{1}$ constituents representing $F_{1}$ and the other of $m_{2}$ constituents representing $F_{2}$. Then $h_{30}$ is the number of triples of constituents lying in different rows and columns of the first group; $h_{03}$ the number of similar triples in the second group; $h_{21}$ the number of triples each consisting of a pair of constituents in the first group and one constituent in the second; $h_{12}$ the number of triples each consisting of one in the first and two in the second. Evidently $H$ is the sum of all the $h_{i j}$, that is 
(2) $\left(m_{1}+m_{2}\right)(\mu-1)(\mu-2)(\nu-1)(\nu-2)$

$$
=6\left(h_{30}+h_{21}+h_{12}+h_{03}\right) \text {. }
$$

From the very nature of the case we have also

$$
\begin{aligned}
& m_{1}(\mu-1)(\mu-2)(\nu-1)(\nu-2)=a_{0} h_{30}+a_{1} h_{21}+b h_{11}, \\
& m_{2}(\mu-1)(\mu-2)(\nu-1)(\nu-2)=a_{0} h_{03}+a_{1} h_{12}+b h_{11},
\end{aligned}
$$

and

(4) $\left(m_{1}-m_{2}\right)(\mu-1)(\mu-2)(\nu-1)(\nu-2)$

$$
=a_{0}\left(h_{30}-h_{03}\right)+a_{1}\left(h_{21}-h_{12}\right),
$$

where $a_{0}, a_{1}$ are numerical constants, $b$ is a function of $\mu$ and $\nu$, and $h_{11}$ is the order of the cone of lines through a given point meeting $F_{1}$ and $F_{2}$ each once, or the number of apparent intersections of the sections of $F_{1}$ and $F_{2}$ by an $S_{3}$. The values of $a_{0}, a_{1}$, being independent of $\mu$ and $\nu$, can be determined without difficulty. If we put $m_{2}=0$, and consequently $m_{1}=\mu \nu, h_{03}=h_{21}=h_{12}=0$ in (4), we have $h_{30}=H$ and $a_{0}=6$. To determine $a_{1}$ let $F_{2}$ be of order $\mu$, represented by a row of constituents in (A). Then $m_{1}=\mu \nu-\mu, m_{2}=\mu, h_{30}=\mu(\mu-1)(\mu-2)$ $\cdot(\nu-1)(\nu-2)(\nu-3) / 6, \quad h_{21}=\mu(\mu-1)(\mu-2)(\nu-1)(\nu-2) / 2$, $h_{03}=h_{12}=0$. Substituting in (4), we find $a_{1}=2$. To determine $b$, it is only necessary to make $m_{2}=1$. Then $h_{30}=h_{12}=0$ and $h_{11}=(\mu-1)(\nu-1)$. Substituting in the second of (3), we obtain $b=(\mu-2)(\nu-2)$. Then (3) and (4) become

$$
\begin{aligned}
& m_{1}(\mu-1)(\mu-2)(\nu-1)(\nu-2) \\
& =6 h_{30}+2 h_{21}+(\mu-2)(\nu-2) h_{11}, \\
& \begin{aligned}
m_{2}(\mu-1)(\mu-2)(\nu & -1)(\nu-2) \\
& =6 h_{03}+2 h_{12}+(\mu-2)(\nu-2) h_{11},
\end{aligned}
\end{aligned}
$$

and

$$
\begin{aligned}
\left(m_{1}-m_{2}\right)(\mu-1)(\mu-2) & (\nu-1)(\nu-2) \\
& =6\left(h_{30}-h_{03}\right)+2\left(h_{21}-h_{12}\right) .
\end{aligned}
$$

From (2) and (5) we obtain

$$
2\left(h_{21}+h_{12}\right)=(\mu-2)(\nu-2) h_{11},
$$


(8) $\left(m_{1}+m_{2}\right)(\mu-1)(\mu-2)(\nu-1)(\nu-2)$

$$
=6\left(h_{30}+h_{03}\right)+3(\mu-2)(\nu-2) h_{11} \text {. }
$$

From (5), (7), (8) one can calculate the $h$ 's if any two of them are known. From the divided array representing the degenerate $F$ it is not difficult to obtain the values of two of the h's. Take a simple illustration. Let $\mu=\nu=3$ and $m_{1}=6, m_{2}=3$. If $F_{1}$ is symbolized by

$\begin{array}{ccc}\alpha_{1} \beta_{1} & \alpha_{2} \beta_{1} & \alpha_{3} \beta_{1} \\ \alpha_{1} \beta_{2} & \alpha_{2} \beta_{2} & . \\ \alpha_{1} \beta_{3} & \cdot & .\end{array}$

and $F_{2}$ by

$$
\begin{array}{cc}
\cdot & \alpha_{3} \beta_{2} \\
\alpha_{2} \beta_{3} & \alpha_{3} \beta_{3}
\end{array}
$$

then we have, by inspection, $h_{30}=1, h_{03}=0$. Either from the formulas or by further inspection we see that $h_{11}=10$, $h_{21}=4, h_{12}=1$.

It is to be added that the method outlined above, applied to $r$-space, enables us to show that the number of apparent $(r-1)$-fold points of an $(r-2)$-dimensional variety which is the intersection of two hypersurfaces in $S_{r}$ of orders $\mu$ and $\nu$ respectively is

$$
H_{r-1}=(r-1) !\left(\begin{array}{c}
\mu \\
r-1
\end{array}\right)\left(\begin{array}{c}
\nu \\
r-1
\end{array}\right) .
$$

The same process of reasoning yields the following formulas analogous to (5) and (6):

$$
\begin{aligned}
& \frac{m_{1}(\mu-1) !(\nu-1) !}{(\mu-r+1) !(\nu-r+1) !}=\sum_{i=0}^{t-1} a_{i} h_{r-i-1, i}+\sum_{j=1}^{t} b_{j} h_{j i} \\
& \frac{m_{2}(\mu-1) !(\nu-1) !}{(\mu-r+1) !(\nu-r+1) !}=\sum_{i=0}^{t-1} a_{i} h_{i, r-i-1}+\sum_{j=1}^{t} b_{j} h_{j i} \\
& \frac{\left(m_{1}-m_{2}\right)(\mu-1) !(\nu-1) !}{(\mu-r+1) !(\nu-r+1) !}=\sum_{i=0}^{t-1} a_{i}\left(h_{r-i-1, i}-h_{i, r-i-1}\right)
\end{aligned}
$$


where $t=(r-1) / 2$ if $r$ is odd and $(r-2) / 2$ if $r$ is even. There is no difficulty in calculating

$$
a_{i}=(r-2) !(r-2 i-1),
$$

but some difficulty is encountered in calculating $b_{j}$ which are functions of $\mu$ and $\nu$. The following are some of their values:

$$
\begin{aligned}
& b_{1}=\quad(\mu-2) !(\nu-2) ! / D, \\
& b_{2}=\quad 4(\mu-4) !(\nu-4) ! / D, \\
& b_{3}=\quad 72(\mu-6) !(\nu-6) ! / D, \\
& b_{4}=2880(\mu-8) !(\nu-8) ! / D, \quad \text { etc. }
\end{aligned}
$$

where $D=(\mu-r+1) !(\nu-r+1)$ !

For $r=3, a_{0}=2, b_{1}=1$ as is well known.* For $r=4, a_{0}=6$, $\dot{a}_{1}=2, b_{1}=b=(\mu-2)(\nu-2)$ as we have seen above.

The University of California

* Salmon, Analytic Geometry of Three Dimensions, 5th ed., vol. 1, pp. 357,358 . 